\title{
The underestimated incidence of an orbital angioleiomyoma is possibly associated with an orbital cavernous hemangioma: illustrative case
}

\author{
Seonah Choi, MD, ${ }^{1}$ JaeSang Ko, MD, $\mathrm{PhD},{ }^{2}$ Se Hoon Kim, MD, PhD, ${ }^{3,4}$ and Eui Hyun Kim, MD, $\mathrm{PhD}^{1,4}$ \\ Departments of ${ }^{1}$ Neurosurgery and ${ }^{3}$ Pathology, Yonsei University College of Medicine, Seoul, Korea; ${ }^{2}$ Department of Ophthalmology, Severance Hospital, Institute of Vision \\ Research, Yonsei University College of Medicine, Seoul, Korea; and ${ }^{4}$ Brain Tumor Center, Severance Hospital, Seoul, Korea
}

\begin{abstract}
BACKGROUND Orbital angioleiomyoma is generally considered a rare tumor; approximately 40 cases have been reported. However, after their experience with 6 consecutive cases in their single institution during 3 years, the authors speculate that the incidence of orbital angioleiomyomas is possibly underestimated.

OBSERVATIONS A 34-year-old female presented with progressive exophthalmos of 2 years' duration. Orbital computed tomography and magnetic resonance imaging revealed a well-circumscribed orbital tumor with partial and heterogeneous gadolinium enhancement. Technetium-99m red blood cell single-photon emission computed tomography showed positive perfusion in the late blood-pool phase, which was exactly consistent with the finding of a cavernous hemangioma. Under the impression of a cavernous hemangioma, the authors accessed the mass with an endoscopic endonasal approach and completely removed it without neurological deficit. Pathological examination revealed that the final diagnosis was an angioleiomyoma with positive immunostaining results for smooth muscle actin (SMA).

LESSONS The incidence of orbital angioleiomyomas may not be very low, as these lesions have possibly been misdiagnosed as orbital cavernous hemangiomas because of their histological similarity. Preoperative presumption and differentiation from cavernous hemangiomas are very challenging because of the rarity of orbital angioleiomyoma and similar radiological findings. SMA immunostaining may be critical to differentiate orbital angioleiomyomas from cavernous hemangiomas.
\end{abstract}

https://thejns.org/doi/abs/10.3171/CASE2172

KEYWORDS angioleiomyoma; cavernous hemangioma; orbit tumor; smooth muscle actin

Angioleiomyoma, also known as vascular leiomyoma or hemangioleiomyoma, is a benign tumor that frequently occurs in the lower extremities. It originates from vascular smooth muscle in the tunica media layer of the vascular wall and is characterized by proliferating pathological vessels containing abundant smooth muscle cells. Most cases develop in the lower extremities; they are rarely reported in the head and neck regions, especially in the orbit. ${ }^{1}$ Since we reported our first case of an orbital angioleiomyo$\mathrm{ma}^{2}{ }^{2}$ we have encountered five more cases of pathologically confirmed orbital angioleiomyomas during the last 3 years, which led us to speculate that the incidence may be higher than reported. Herein, we report a case of an orbital angioleiomyoma that mimicked an orbital hemangioma and present an in-depth discussion on its differential diagnosis through a literature review.

\section{Illustrative Case}

\section{Patient History and Examinations}

A 34-year-old female presented with progressive exophthalmos of 2 years' duration. Her visual acuity was decreased on her left side. Tests for extraocular muscles revealed no particular restriction or pain, although she complained of subjective diplopia on her left gaze. Her Hess screen chart results showed a minimal difference in the left inferior oblique muscle. Ocular fundoscopic examination revealed left papilledema (Fig. 1), which was consistent with increased

ABBREVIATIONS EEA = endoscopic endonasal approach; IHC = immunohistochemistry; MRI = magnetic resonance imaging; OCT = optical coherence tomography; $\mathrm{RBC}=$ red blood cell; RNFL = retinal nerve fiber layer; SMA = smooth muscle actin; SPECT = single-photon emission computed tomography; Tc-99m = technetium-99m. INCLUDE WHEN CITING Published May 24, 2021; DOI: 10.3171/CASE2172.

SUBMITTED February 3, 2021. ACCEPTED March 3, 2021.

(C) 2021 The authors, CC BY-NC-ND 4.0 (http://creativecommons.org/licenses/by-nc-nd/4.0/). 

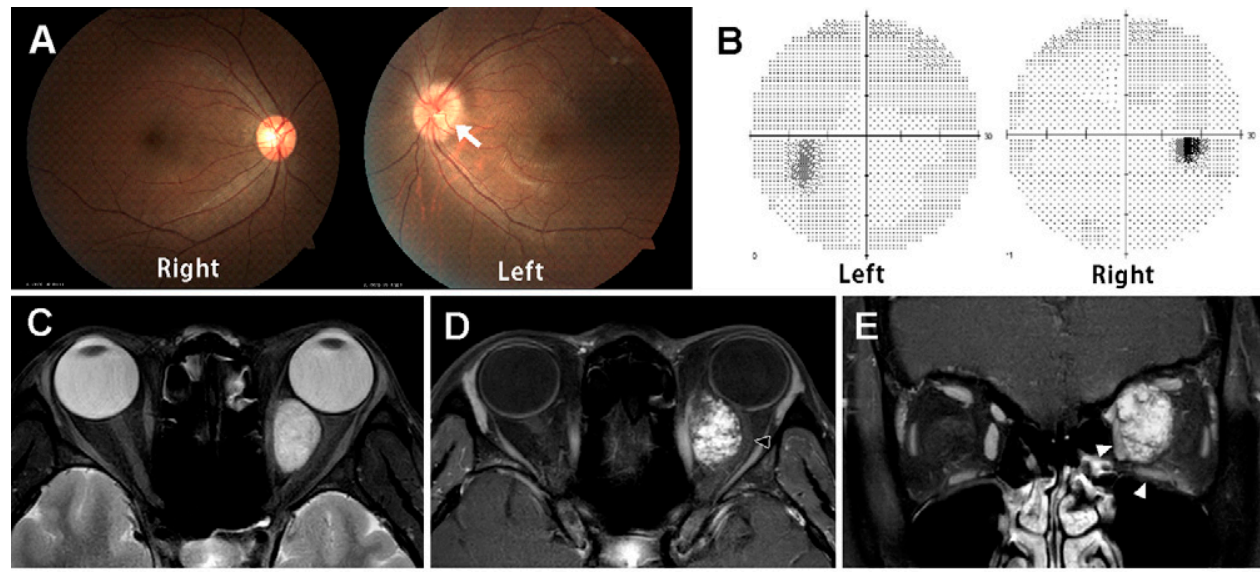

$\mathbf{F}$

G
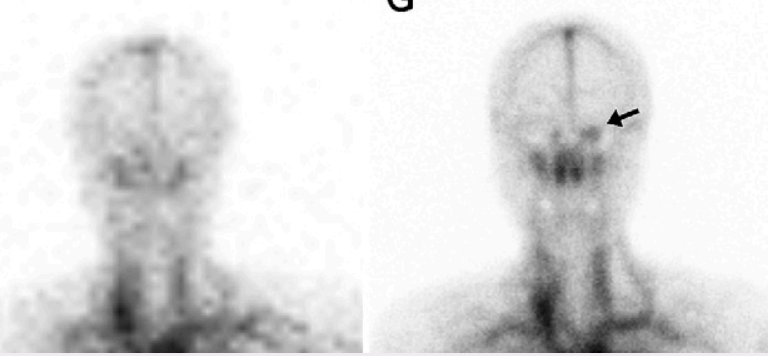

H

FIG. 1. Preoperative examinations. A: Ocular fundoscopic examination revealed papilledema (white arrow) on the left side optic disc. B: Perimetry test showed no significant visual field defect on both sides. MRI demonstrated a round tumor with homogeneous hyperintensity on a T2-weighted image $(\mathbf{C})$, with heterogeneous gadolinium enhancement on a T1-weighted image (D), and compressing the optic nerve laterally (black arrowhead). E: The relationship between tumor and medial/inferior rectus muscles (white arrowheads) was noticed on the coronal T1-weighted gadolinium-enhanced image. Tc-99m RBC scan with perfusion (F), early blood pool (G), and late blood pool $(\mathbf{H})$ images shows perfusion/blood pool mismatch (black arrows).

retinal nerve fiber layer (RNFL) thickness on optical coherence tomography $(\mathrm{OCT})$.

Orbital computed tomography showed a round, well-circumscribed, space-occupying intraconal mass with iso- to hyperdensity compressing the left optic nerve. Magnetic resonance imaging (MRI) also demonstrated a partially and heterogeneously gadolinium-enhanced round mass just lateral to the left medial rectus muscle on T1-weighted images; relatively homogeneous hyperintensity was observed on T2-weighted images. Suspecting an orbital cavernous hemangioma, which is the most common benign mass-forming lesion in the orbit, we performed technetium (Tc)-99m red blood cell (RBC) single-photon emission computed tomography (SPECT), as it is highly useful in the diagnosis of this condition. ${ }^{3,4}$ We observed a perfusion/ blood pool mismatch with negative uptake in the perfusion phase, weak positive uptake in the early blood-pooling phase, and positive uptake in the late blood-pooling phase, which was exactly consistent with a cavernous hemangioma. Considering the location of the tumor inside the orbit and its relative anatomical orientation with the optic nerve, resection through an endoscopic endonasal approach (EEA) was planned.

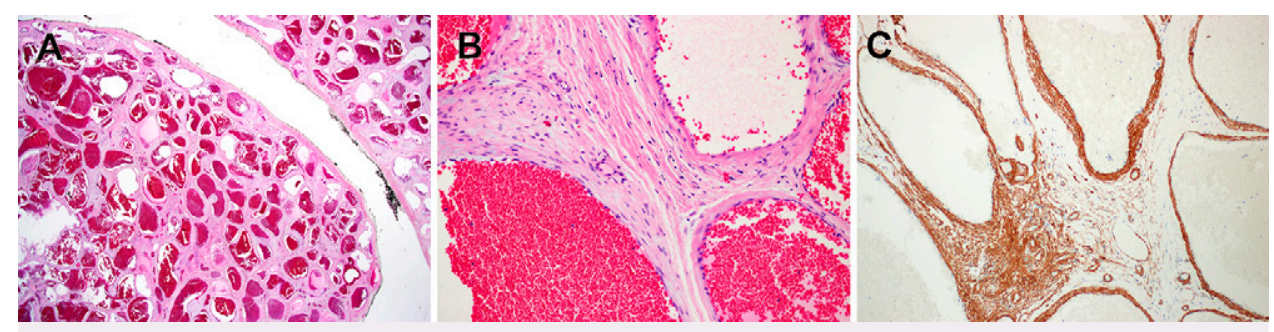

FIG. 2. Histopathological examination. A well-demarcated mass is demonstrated with multiple enlarged vascular channels with partially thickened vessel walls $(\mathbf{A}$; hematoxylin and eosin [H\&E], original magnification $\times 40)$. Fibrous stroma surrounding a vascular channel $(B ; H \& E$, original magnification $\times 400)$ with prominent smooth muscle cells $(\mathbf{C}$; SMA, original magnification $\times 100)$ is noted. 


\section{Operation and Surgical Findings}

The EEA to the left orbital tumor was simulated preoperatively, and resection via the EEA was considered feasible. A rigid endoscope 4 $\mathrm{mm}$ in diameter and $18 \mathrm{~cm}$ in length (Synergy UHD4 endoscope system, Arthrex) and a cranial navigation system (StealthStation S8 surgical navigation system, Medtronic) were used during surgery. A bilateral nostril, four-handed EEA was employed to access the left orbital tumor. First, a hemitransfixion incision (also known as Freer's incision) was made on the medial mucocutaneous junction on the septum, just behind the right nostril. The right septal mucosa was reflected laterally, and the perpendicular plate of the ethmoid and a superior part of the vomer were removed for later use in reconstruction at the very last stage of the operation. A transverse incision was made on the left septal mucosa to secure transseptal accessibility from the right side of the nostril to the left orbit. After a left middle turbinectomy, a posterior ethmoidectomy was performed with removal of the uncinated process and ethmoid bullae until we exposed the optic canal and medial wall of the orbit (lamina papyracea). After removal of the lamina papyracea, a careful periorbital incision was made to avoid intraorbital structure injury. After localizing the tumor with the aid of the neuronavigation system, the space between the medial and inferior rectus muscles was dissected. Fat tissue was partially removed and pushed aside with cottonoid. After intraorbital dissection, the tumor was easily identified as a solid, pinkish, hypervascularized mass. The tumor capsule was not adhered to the surrounding structures, which facilitated extracapsular dissection. Electrooculographic monitoring was continued during dissection to prevent injury to the oculomotor nerve and its branches. ${ }^{5}$ After cauterization of a few feeding arteries, the tumor was removed in one piece. After bleeding control was achieved, the periorbital layer was closed with several stay sutures and covered with a fleece-coated fibrin glue patch (TachoSil, Nycomed Austria). The medial orbital wall was reconstructed with placement of the septal bone piece, which was reinforced by a topical tissue sealant (BioGlue, CryoLife Inc.) (Video 1).

VIDEO 1. Case illustration of EEA for a left orbital tumor. The 34year-old female patient with left proptosis presented with a medial orbital tumor, confirmed as angioleiomyoma later. For the best performance, we chose the binostril EEA. The middle turbinate and posterior ethmoid sinus were removed on the tumor side. Freer's incision was made on the opposite nostril, and we reflected septal mucosa. We tried to take nasal bone in a piece to be used for reconstruction later. After the bone was removed, we made another incision on the opposite side of septal mucosa so that tumor was approached from the contralateral nostril though the septum. The sphenoid and maxillary sinuses were also opened for surgical freedom. We removed the medial orbital wall from the very proximal side of the view to near the apex. With navigation guidance, the tumor's location was confirmed, and we split the periorbita several ways and removed some of the fat. Then we performed careful dissection between the medial and inferior rectus muscles. We found the highly vascularized, solid mass with several feeding arteries on its medial side. These vessels were carefully dissected and coagulated, and these were continued until we freed the tumor from the surrounding intraconal compartments. Since there was less adhesion on its lateral side, the tumor was removed easily in one piece. To prevent possible injury on the oculomotor nerve and its branches, electrooculographic monitoring was continued during the procedure. After approximating the periorbital layer, part of the nasal bone was used for orbital wall reconstruction and covered with a fibrin sealant patch and glue. The septal mucosa was closed with nasal bone reconstruction. Postoperative MRI confirmed total resection with improved proptosis. Click here to view.

\section{Pathological Findings}

Hematoxylin and eosin staining of the tumor revealed multiple enlarged vascular channels with partially thickened vessel walls containing spindle-shaped tumor cells. Immunohistochemistry $(\mathrm{IHC})$ revealed a strong positivity for smooth muscle actin (SMA) in the tumor, consistent with an angioleiomyoma (Fig. 2).

\section{Postoperative Course}

Postoperative MRI confirmed complete resection (Fig. 3). The patient's exophthalmos fully resolved immediately after the operation, and no postoperative neurological deficits developed. The subjective diplopia symptom was also resolved. Postoperative OCT demonstrated a decrease in RNFL thickness, and fundoscopic examination showed complete resolution of papilledema.
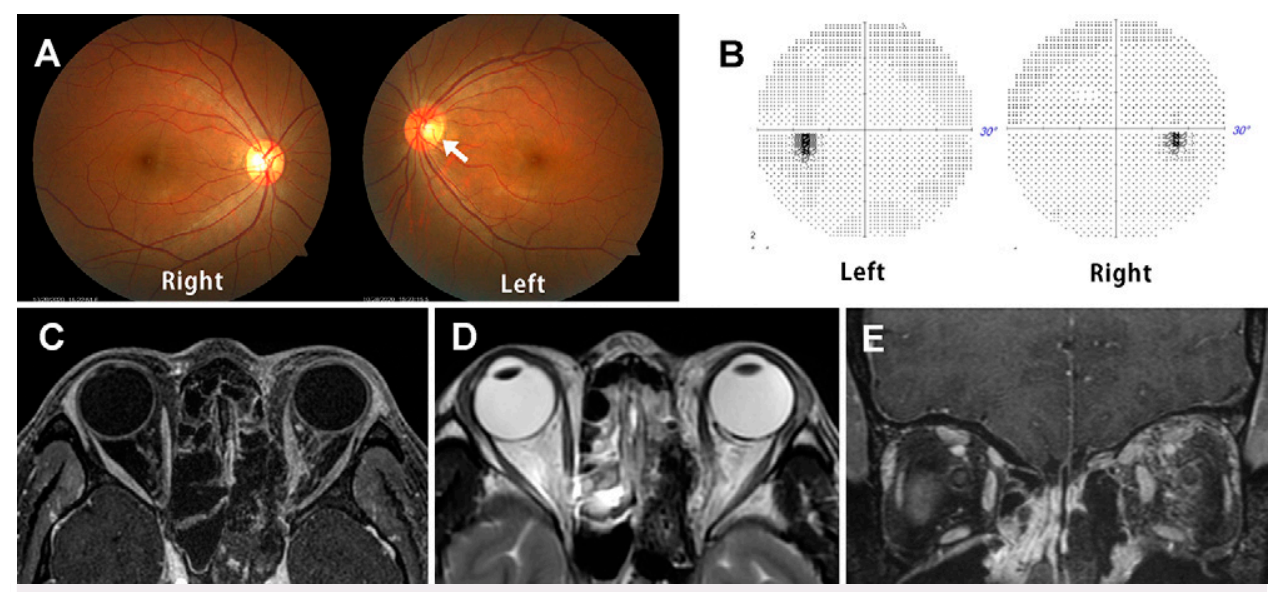

FIG. 3. Postoperative examinations. A: Papilledema was resolved on 1-month follow-up fundoscopic examination (white arrow). B: Visual field test showed no neurological deficit postoperatively. C-E: MRI with T1weighted gadolinium-enhanced images and T2-weighted image confirmed complete removal of the tumor. 


\section{Single-Institution Experience of Orbital Angioleiomyomas}

Since our first patient with orbital angioleiomyoma, ${ }^{2}$ we have encountered a total of six cases of pathologically confirmed orbital angioleiomyomas at a single institution over 3 years. Four orbital angioleiomyomas were relatively small in size, located at the apex with decreased visual acuity as the main symptom. The other two were large tumors with profound exophthalmos. Interestingly, two were evaluated with Tc-99m RBC SPECT, which showed evident perfusion/blood pool mismatches. Three patients underwent transcranial surgery with frontotemporal craniotomy, and the other three tumors were approached via EEA. Total removal was achieved in all six cases, and none experienced long-standing neurological complications. The details of our six patients are summarized in Table 1.

\section{Discussion}

\section{Observations}

Angioleiomyoma is a benign tumor originating from vascular smooth cells and synonymous with vascular angioleiomyoma, hemangioleiomyoma, angiomyoma, or dermal angioma. ${ }^{6}$ It is a rare soft tissue tumor with the highest prevalence in middle-aged females, and most affect the lower extremities. ${ }^{7}$ Angioleiomyoma as an orbital/periorbital lesion is much rarer, with only 40 cases of pathologically proven orbital angioleiomyomas reported in the literature so far. ${ }^{2,8,9}$ Our single-institution experience of six cases during such a short period cast doubt on the extremely low incidence of orbital angioleiomyoma. There were several attempts to obtain a large-scale histopathological classification of the orbital tumors in recent decades. Most of the classifications included thousands of orbital/periorbital lesions, and the top-ranked benign tumors were either cavernous hemangiomas or dermoid cysts. ${ }^{10-12}$ However, one interesting study with the largest patient population reported similar incidence rates for cavernous hemangioma and vascular leiomyoma among the orbital lesions. ${ }^{13}$ We therefore assumed that the incidence of angioleiomyoma, which is extremely low in the literature, might be much higher than reported.

According to the report by Morimoto, ${ }^{7}$ angioleiomyomas have three histological subtypes of solid, venous, and cavernous, and they can be divided into two groups based on location and populations. The larger group comprises solid, painful masses in the extremities, and the smaller group presents with venous, painless masses in the head. The three subtypes are determined by quantitative differences between vascular channels and smooth muscle cells, with the lowest incidence of the cavernous type in the extremities. ${ }^{14}$ Conversely, as including in the orbit. ${ }^{9}$ This is very interesting because the cavernous type is characterized by the fewest smooth muscle cells and the most abundant pathological vascular channels, sharing histological similarities with cavernous hemangiomas.

Although preoperative presumption of an angioleiomyoma based only on clinical and radiological findings is very challenging, there are studies suggesting radiological characteristics that help guide the differential diagnosis. ${ }^{6}$ Tumors with flame-like partial enhancement on T1-weighted gadolinium-enhanced imaging ${ }^{16}$ and delayed progressive enhancement on dynamic $\mathrm{MRI}^{17}$ were suggested as characteristics of an angioleiomyoma. TC-99m RBC SPECT, also known as RBC scintigraphy or RBC scan, has a high predictive value for diagnosing orbital cavernous hemangioma. ${ }^{3,4,18}$ However, both cases of angioleiomyoma evaluated by RBC scan in our series showed the same findings indicative of cavernous hemangioma. an intracranial lesion, the cavernous type was the most frequent, ${ }^{15}$

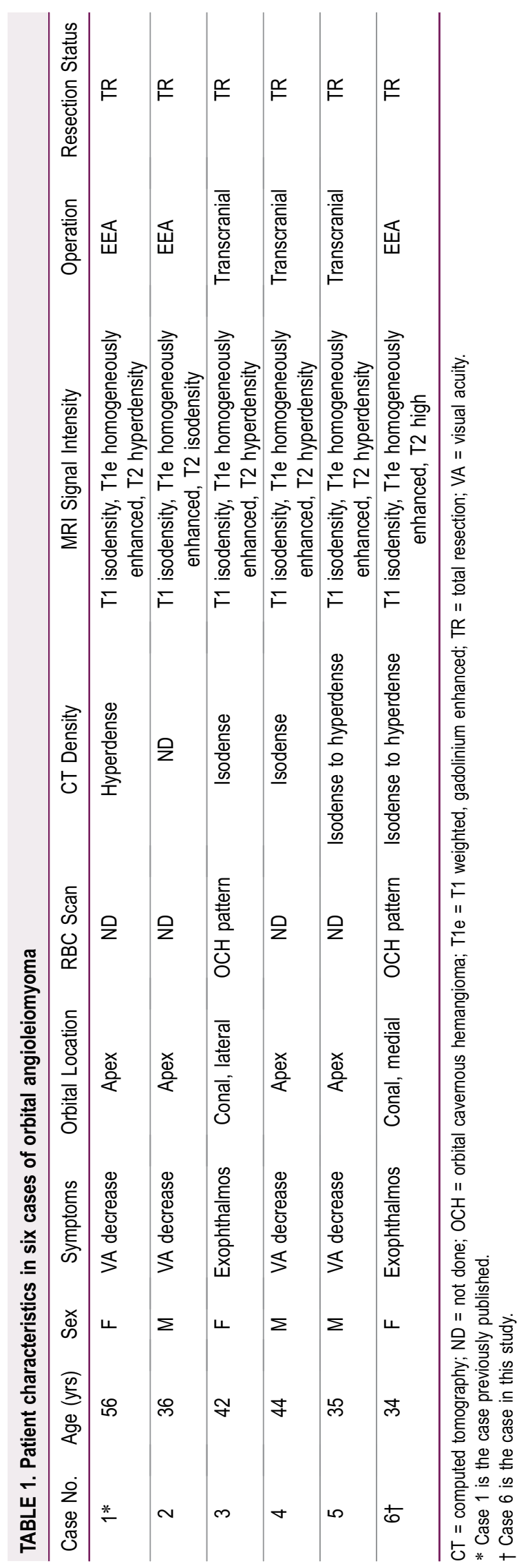

4 | J Neurosurg Case Lessons | Vol 1 | Issue 21 | May 24, 2021 
Considering the histological similarities, it is possible that the cavernous type of an orbital angioleiomyoma mimics a cavernous hemangioma on MRI and RBC scans and may be misdiagnosed, especially when the diagnosis is not based on histopathological examination. This could explain why the incidence of orbital angioleiomyomas has been underestimated. Although angioleiomyoma is known to be radioresistant and resection is the treatment of choice, ${ }^{19}$ it is possible that the cavernous subtype of orbital angioleiomyoma shows a good response to radiation similar to that of cavernous hemangioma. We recommend performing $\mathrm{IHC}$ for SMA in every case of orbital cavernous hemangioma. Although the differentiation between a cavernous subtype of angioleiomyoma and a cavernous hemangioma is not always easy, the presence of smooth muscle cells and the amount and thickness of the smooth muscle layer will be strong evidence of an angioleiomyoma. Our hypothesis can be tested by retrospective SMA IHC on tissues previously diagnosed as cavernous hemangiomas.

\section{Lessons}

The incidence of orbital angioleiomyoma may have been underestimated because of its histological similarity with orbital cavernous hemangioma. Preoperative differentiation from cavernous hemangiomas is very challenging because of the rarity of orbital angioleiomyoma and similar radiological findings. We recommend that SMA immunostaining be performed during differential diagnosis.

\section{Acknowledgments}

This work was funded by Yonsei University College of Medicine (6-2020-0224).

\section{References}

1. Sung MS, Lee MJ, Kim NJ, et al. A case of orbital angioleiomyoma. J Korean Ophthalmol Soc. 2008;49(4):673-676.

2. Lee $B$, Park SJ, Moon JH, et al. Angioleiomyoma in the orbital apex: a case report. Brain Tumor Res Treat. 2019;7(2):156-159.

3. Polito E, Burroni L, Pichierri P, et al. Technetium Tc $99 \mathrm{~m}$-labeled red blood cells in the preoperative diagnosis of cavernous hemangioma and other vascular orbital tumors. Arch Ophthalmol. 2005;123(12): 1678-1683.

4. Burroni L, Borsari G, Pichierri $P$, et al. Preoperative diagnosis of orbital cavernous hemangioma: a ${ }^{99 m}$ Tc-RBC SPECT study. Clin Nucl Med. 2012;37(11):1041-1046.

5. Jeong HN, Ahn SI, Na M, et al. Triggered electrooculography for identification of oculomotor and abducens nerves during skull base surgery. J Korean Neurosurg Soc. 2021;64(2):282-288.

6. Ramesh P, Annapureddy SR, Khan F, Sutaria PD. Angioleiomyoma: a clinical, pathological and radiological review. Int J Clin Pract. 2004;58(6):587-591.

7. Morimoto N. Angioleiomyoma [vascular leiomyoma]-a clinicopathologic study. Med J Kagoshima Univ. 1974;24:663-666.
8. Arat YO, Font RL, Chaudhry IA, Boniuk M. Leiomyoma of the orbit and periocular region: a clinicopathologic study of four cases. Ophthal Plast Reconstr Surg. 2005;21(1):16-22.

9. Wong SW, Laybourne J, Irion L, Cook A. A rare case of orbital angioleiomyoma. Orbit. 2021;40(1):60-64.

10. Ni C, Ma X. Histopathologic classification of 1921 orbital tumors. Article in Chinese. Yan Ke Xue Bao. 1995;11(2):101-104.

11. Bonavolontà $G$, Strianese $D$, Grassi $P$, et al. An analysis of 2,480 space-occupying lesions of the orbit from 1976 to 2011. Ophthal Plast Reconstr Surg. 2013;29(2):79-86.

12. Shields JA, Shields CL, Scartozzi R. Survey of 1264 patients with orbital tumors and simulating lesions: The 2002 Montgomery Lecture, part 1. Ophthalmology. 2004;111(5):997-1008.

13. He Y, Song G, Ding Y. Histopathologic classification of 3476 orbital diseases. Zhonghua Yan Ke Za Zhi. 2002;38(7):396-398.

14. Hachisuga T, Hashimoto H, Enjoji M. Angioleiomyoma. A clinicopathologic reappraisal of 562 cases. Cancer. 1984;54(1):126-130.

15. Li CB, Xie MG, Ma JP, et al. Primary intracranial angioleiomyomas as rare, nonmalignant, and distinct neoplastic entities: a series of 8 cases and a literature review. World Neurosurg. 2018;113:1-13.

16. Sun L, Zhu Y, Wang H. Angioleiomyoma, a rare intracranial tumor: 3 case report and a literature review. World J Surg Oncol. 2014;12:216.

17. Colnat-Coulbois S, Schmitt E, Klein O, et al. Angioleiomyoma of the cavernous sinus: case report. Neurosurgery. 2008;62(1):E257-E258.

18. Gdal-On M, Gelfand YA, Israel O. Tc-99m labeled red blood cells scintigraphy: a diagnostic method for orbital cavernous hemangioma. Eur J Ophthalmol. 1999;9(2):125-129.

19. Jakobiec FA, Howard GM, Rosen M, Wolff M. Leiomyoma and leiomyosarcoma of the orbit. Am J Ophthalmol. 1975;80(6):1028-1042.

\section{Disclosures}

The authors report no conflict of interest concerning the materials or methods used in this study or the findings specified in this paper.

\section{Author Contributions}

Conception and design: EH Kim, Choi, SH Kim. Acquisition of data: all authors. Analysis and interpretation of data: EH Kim, Choi, Ko. Drafting the article: Choi, SH Kim. Critically revising the article: EH Kim.

Reviewed submitted version of manuscript: EH Kim, SH Kim. Approved the final version of the manuscript on behalf of all authors: EH Kim. Statistical analysis: Choi. Administrative/technical/material support: EH Kim. Study supervision: EH Kim.

\section{Supplemental Information \\ Videos}

Video 1. https://vimeo.com/519547976.

\section{Correspondence}

Eui Hyun Kim: Yonsei University College of Medicine, Seoul, Republic of Korea. euihyunkim@yuhs.ac. 